\title{
Microfluidic reprogramming to pluripotency of human somatic cells
}

Onelia Gagliano ${ }^{1,2,3,{ }^{*}}$, Camilla Luni ${ }^{1, *}$, Wei Qin ${ }^{1,4,5}$, Enrica Bertin ${ }^{3}$, Erika Torchio ${ }^{1,2,3}$, Silvia Galvanin $^{1,2,3}$, Anna Urciuolo ${ }^{2,3} \&$ Nicola Elvassore ${ }^{1,2,3,6, \#}$

${ }^{1}$ Shanghai Institute for Advanced Immunochemical Studies (SIAIS), ShanghaiTech University, Shanghai, China

${ }^{2}$ Department of Industrial Engineering, University of Padova, Padova, Italy

${ }^{3}$ Venetian Institute of Molecular Medicine, Padova, Italy

${ }^{4}$ Institute of Biochemistry and Cell Biology, Shanghai Institutes for Biological Sciences, Chinese Academy of Sciences, Shanghai, China

${ }^{5}$ University of Chinese Academy of Sciences, Beijing, China

${ }^{6}$ Stem Cells \& Regenerative Medicine Section, UCL Great Ormond Street Institute of Child Health, London, UK

${ }^{*}$ These authors contributed equally to this work.

Email addresses:

Onelia Gagliano: onelia.gagliano@unipd.it

Camilla Luni: camilla.luni@shanghaitech.edu.cn

Wei Qin: qinwei@shanghaitech.edu.cn

Enrica Bertin: enrica.bertin@gmail.com

Erika Torchio: erika.torchio@studenti.unipd.it

Silvia Galvanin: silvia.galvanin@studenti.unipd.it

Anna Urciuolo: anna.urciuolo@unipd.it

${ }^{\#}$ Corresponding author:

Nicola Elvassore

E-mail: n.elvassore@ucl.ac.uk

Stem Cells \& Regenerative Medicine Section, UCL Great Ormond Street Institute of Child Health, London, UK

Telephone: +39049 8275469

Fax: +39049827 7599

KEYWORDS pluripotency, stem cell, microfluidics, reprogramming, iPSC, induced pluripotent stem cell, mRNA 


\begin{abstract}
Induced pluripotent stem cells (iPSCs) have a number of potential applications in stem cell biology and regenerative medicine, including precision medicine. However, their potential clinical application is hampered by the low efficiency, high costs, and intensive workload of the reprogramming process. Here, we describe a protocol to reprogram human somatic cells to hiPSCs with high efficiency in 15 days using microfluidics. We successfully downscaled an 8day protocol based on daily transfections of mRNA encoding for reprogramming factors and immune evasion proteins. Using this protocol, we obtain hiPSC colonies (up to $160 \pm 20$ in a single $27-\mathrm{mm}^{2}$ microfluidic chamber) 15 days after seeding $\sim 1500$ cells per independent chamber and under xeno-free defined conditions. Only $\sim 20-\mu \mathrm{L}$ medium is required per day. The hiPSC colonies extracted from the microfluidic chamber do not require further stabilization due to the short lifetime of mRNA. The high success rate of reprogramming in microfluidics, under completely defined conditions, enables hundreds of cells to be simultaneously reprogrammed, with an $\sim 100$-fold cost reduction of raw material compared to a standard multiwell culture condition. This system also enables the generation of hiPSCs suitable for clinical translation or further research into the reprogramming process.
\end{abstract}




\section{INTRODUCTION}

The discovery that adult human somatic cells can be reprogrammed to pluripotency ${ }^{1}$ has enabled previously inaccessible processes of human biology to be studied in vitro, and new personalized biomedical applications to be envisioned in vivo.

Somatic cell reprogramming can be induced by the forced expression of POU5F1, SOX2, KLF4 and C-MYC (OSKM, known as Yamanaka's factors) ${ }^{1}$. In the initial stage of reprogramming, the exogenous expression of these transcription factors perturbs the transcriptional network of somatic cells. In response, cells integrate intrinsic and extrinsic cues to remodel chromatin and reach a new epigenetic state. The latter stages of reprogramming, maturation and stabilization, result in the endogenous expression of the core circuitry of pluripotency.

We aimed to develop a protocol for the cost-effective production of quality-controlled human pluripotent stem cells, in non-integrating and chemically defined xeno-free culture conditions. The method we developed enables reprogramming of a limited number of human somatic cells with high efficiency, high throughput, and low costs to produce hiPSCs without genomic integration. ${ }^{2}$ These results were achieved by downscaling mRNA-based cell reprogramming, using microfluidics to miniaturize the system.

\section{Development of the protocol}

Miniaturization results in a $\sim 50$-fold increase of efficiency compared to conventional culture systems, even under defined xeno-free conditions ${ }^{2}$. Miniaturization provides two significant advantages: a substantial improvement in mRNA delivery, as a consequence of the short distance between the synthetic mRNA and the cells ${ }^{2}$; and a faster accumulation of cellular extrinsic endogenous factors that positively feedback on cellular reprogramming (Figure 1a). This self-regulation of cellular microenvironment promotes the conversion of fibroblasts into hiPSC colonies only when a critical ratio between cell density and medium height is used ${ }^{2}$. This protocol utilizes the advantages of mRNA reprogramming in standard systems, such as the rapidity of exogenous mRNA clearance, due to the $\sim 24$ hour lifespan of mRNA in the cells $^{3}$. In microfluidics, after only 15 days of reprogramming, a high number of hiPSC colonies are obtained (up to $160 \pm 20$ hiPSC colonies per culture chamber). These colonies can then be extracted from the setup and used for other applications.

The miniaturization of this protocol also offers specific advantages. First, a low number of cells is required ( $\sim 1600$ cells/chamber). Thus, this protocol can be used when there is limited availability of biological samples, for example, when using primary cells or when cell expansion and cell senescence prevent a successful reprogramming. Second, in a single microfluidic device ten independent parallel experiments can be performed, greatly increasing the experimental throughput. A single operator can easily manage a hundred parallel reprogramming experiments with a normal workload. Third, the small volume of the system $(\sim 5 \mu \mathrm{L})$ reduces the cost of reagents more than 100-fold and has a significant impact on the feasibility of reprogramming on a large scale. Many conditions of reprogramming can be tested for a single cell line to increase the reprogramming success rate, as discussed below; somatic cells from large cohorts of patients can be affordably reprogrammed; and multiparametric optimization becomes possible for the development of integrated processes of reprogramming and differentiation ${ }^{2,4,5,6}$. 
The protocol we describe here has been improved compared to that used in our original publication $^{2}$. Previously, the reprograming was based on a daily transfection of modified mRNAs (OCT4, SOX2, KLF4, C-MYC, NANOG, LIN28 (OSKMNL)) for at least 12 days. Moreover, the transfections were coupled with two changes per day of medium supplemented with $\mathrm{B} 18 \mathrm{R}$ protein, to reduce interferon response. Medium was freshly prepared every day and reprogramming was performed in normoxia. This protocol was highly effective but required an intensive workload during the entire duration of the reprogramming that could last up to 15 days. Thus, we further optimised the protocol to reduce the complexity of the protocols in terms of medium preparation and to reduce the overall number of medium changes and transfections, while maintaining the high efficiency of reprogramming.

The improved version of the protocol we describe here enables microfluidic reprogramming based on commercially available non-modified mRNAs (NM-RNAs) of the same reprogramming factors (OSKMNL) combined with immune evasion NM-RNAs encoded for $E 3, K 3$ and B18R protein $(E K B)$, and two microRNAs (miRNAs) from the 302/367 cluster, previously reported in a published protocol ${ }^{7}$. The reprograming process is performed under hypoxia $\left(5 \% \mathrm{O}_{2}\right)$, an established favorable factor to improve reprogramming efficiency ${ }^{8}$. To efficiently downscale the reprogramming in the microfluidic system, we limited the number of daily transfections to 8 for the entire reprogramming, with a single medium change per day. Moreover, we also adapted the reprogramming protocol to use chemically defined medium (E7 medium). E7 has the same components present in E8 ${ }^{9}$ (the widely used medium for hiPSC expansion) except that TGF $\beta$ is absent. It has a disclosed and simple composition, which makes it suitable for defined biological studies of cell reprogramming. Finally, we used a new method, based on ethylenediaminetetraacetic acid (EDTA) as detachment agent, to selectively extract hiPSC colonies from microfluidic chambers.

\section{Application of the protocol}

Microfluidic reprogramming can be adapted to a wide range of applications. There are no major limitations in downscaling other reprogramming methods to the microfluidic system, after optimizing the conditions. Moreover, given the feasibility of producing customized mRNAs by common biological laboratories, the coupling of microfluidic and mRNA technologies gives great flexibility for other applications.

As an example, we performed fibroblast reprogramming in microfluidics also using a different approach, based on self-replicating RNAs of only four reprogramming factors, OCT-4, KLF4, SOX-2 and GLIS1. The results in Supplementary Figure 1 show that cells can be reprogrammed within this setup also with only three Yamanaka factors and without C-MYC, with a consistent success rate in 20 independent microfluidic chambers.

\section{Comparison with other methods}

In recent years, numerous methods have been developed to generate hiPSCs, aimed at increasing the efficiency of reprogrammed cells and also enabling the generation of footprintfree hiPSC lines that lack integration of any viral vector sequences in their genomes. Replication-competent Sendai RNA virus carrying the Yamanaka factors (OCT4, SOX2, KLF4, c-MYC) is widely used for skin fibroblast reprogramming ${ }^{10}$. It is relatively efficient and highly reliable with a low workload. However, the Sendai virus method does not allow flexibility in the expression level and stoichiometry of the reprogramming factors, and there is relatively slow clearance of Sendai virus (more than 10 passages ${ }^{11,12}$ ). The substantial amount 
of work required to clear virus from the hiPSCs during expansion results in having high costs for hiPSC colony stabilization. Episomal plasmids, which can be easily produced by single biological laboratories and provide process flexibility ${ }^{13}$, have been shown to have lower efficiency compared to Sendai virus, and the generated hiPSCs retain episomal plasmid sequences at high passages $(>10)$, and have a high aneuploidy rate. Alternatively, a selfreplicating RNA-based method has also been proposed ${ }^{14}$, but the complete clearance dynamics of the exogenous RNA, which is removed from the cells by the innate immune response, has not been investigated in a comparative study. These features discourage the use of these methods for clinical applications which require hiPSCs of high quality.

Using mRNA technology for reprogramming ${ }^{15,16}$ has advantages over other non-integrating reprogramming methods. Efficiency is up to $4 \%$, and colonies emerge faster than with other methods. The aneuploidy rate is low, and there is a complete absence of cellular integration after less than 5 passages ${ }^{11}$. Moreover, mRNA can be easily produced by biological laboratories, increasing the flexibility of mRNA mix composition, stoichiometry and level of expression. Specialized companies have already achieved clinical-grade mRNA transfection systems. As also reported in a recent benchmark study that compared the pros and cons of non-integrating methods ${ }^{11}$, reprogramming with mRNAs is the most promising approach to fully exploit the potential of hiPSC technology in clinical applications and research settings.

All methods for hiPSC derivation based on conventional culture systems (either Petri dish or multiwell) have considerably high costs, and this can be a limiting factor for many laboratories, especially when multiple patient samples need to be reprogrammed. For example, amongst the most popular integration-free methods, Sendai virus and mRNA approaches need expensive reagents for reprogramming. Moreover, high labor costs are required due to intensive expansion of either starting cells, when low efficient methods are used, or hiPSCs, when dilution and clearance of exogenous materials is necessary. For all these reasons, many researchers are limited to small numbers of simultaneous reprogramming, which are often not sufficient to satisfy the need for multiple controls and multiple patient samples. For example, a six-sample hiPSC project, including 3 controls and 3 patients, costs more than 10,000 euro in reagents and requires one full-time researcher to generate hiPSCs. Characterization and clone selection increases costs further. In the best scenario, a recent comparative study showed that the highest efficiency of non-integrating methods is approximately $4 \%$ when using $\mathrm{mRNA}^{11}$.

Microfluidic reprogramming enables large-scale and high-efficiency production of high quality hiPSCs. Microfluidic technology substantially decreases the cost of reagents and provides the highest efficiency of reprogramming methods. Using microfluidic reprogramming we were able to generate an average number of 6 colony $/ \mathrm{mm}^{2}$ in 15 days, which is higher than other methods reported in the literature, which range between 0.1 and 4 colony/mm $\mathrm{mm}^{2}$ in 6 -well plate ${ }^{17}$. Microfluidic reprogramming requires the seeding of a low number of cells, thus there is a high probability of obtaining colonies. Reprogrammingresistant cells can also be reprogrammed ${ }^{2}$. In addition, the cost of reagents can be decreased by $\sim 100$ fold as microfluidic platforms require only few microliters of medium (a single chamber is $\sim 5 \mu \mathrm{L}$ ), and only few nanograms of mRNA is required per day compared to almost $2 \mu \mathrm{g}$ when using standard conditions. Lastly, microfluidics is amenable to automation, 
offering the possibility of manipulating the microenvironment in a high precision manner with remote control, strongly reducing the workload and increasing the experimental throughput. Automation is important for large-scale production under strictly defined and clinical grade conditions. Recently, consortia have been formed to increase the availability of hiPS cell lines, such as the Human Induced Pluripotent Stem Cells Initiative (HipSci, http://www.hipsci.org) and the iPSC Repository of the California Institute for Regenerative Medicine (CIRM, https://www.cirm.ca.gov/researchers/ipsc-repository). Cost reduction coupled with system automation make possible to perform hundreds of parallel reprogramming experiments from different cell sources. Currently, even in manual mode, a single operator can easily manage a hundred samples in parallel, using a greatly reduced amount of reagents.

\section{Limitations}

Reprogramming different cell lines results in different success rates. This is a recognized problem in the field and we have also experienced this limitation ${ }^{2}$. The reasons behind these differences can be ascribed to differences in primary fibroblast isolation protocols, in fibroblast senescence and tissue of origin, in patient age and other patient-specific factors. While understanding the origin of this variability is still complicated, and the absolute reprogramming efficiency very variable, we found that microfluidics shows a great improvement in both success rate and number of colonies per cell seeded when compared to standard well culture systems. In the troubleshooting section, adaptations of the protocol to increase the success rate based on specific cell line behavior are discussed, but we recommend to run different reprogramming conditions in parallel.

The small number of cells that is possible to culture on each microfluidic chamber surface (whose area is typically $<30 \mathrm{~mm}^{2}$ ) could be limiting for methods of analyses that require a high number of cells, such as transcriptomic and proteomic characterizations. Pooled samples and the use of single-cell technologies can overcome this limitation.

mRNA reprogramming is labor-intensive because of the need for daily transfections. However there is a quick clearance of exogenous materials from the obtained hiPSCs. Using already developed automation could further assist in protocol development and increase throughput ${ }^{18}$.

\section{Experimental Design}

The workflow shown in Figure 2 describes the whole protocol (Box 1, Box 2 and Steps 1-27). Four main tasks need to be undertaken, as discussed below.

Microfabrication. Box 1 details the procedure for microfluidic device fabrication. A "Mask design" is provided in the Supplementary Manual, whose layout is shown in Supplementary Figure 2. Thus, a design stage is not needed, unless modifications to the proposed configuration are required. The "Master mold fabrication" is performed according to standard photolithographic techniques. It needs to be performed only once, as the master mold can be re-used several times. If facilities are not available, it is advisable to outsource it, providing an external company with the photomask. "Microfluidic device production" refers to the standard soft-lithographic techniques used for the production of the microfluidic devices, called chips, made of polydimethylsiloxane (PDMS), and its attachment to a glass slide to build the final 
device. This step can be outsourced. Companies, such as Onyel Biotech (Italy) or any other company that sells PDMS chips for long-term cell culture, can provide the microfluidic chip according to the protocol described in Box 1. We refer the reader to Box 1 and a previous protoco $l^{19}$ for instructions on how to perform these first three parts. Final sterilization of the microfluidic devices can be performed by standard autoclaving.

Fibroblast culture in microfluidics. Advice on how to perform cell culture within microfluidic culture chambers is given. Before proceeding with a reprogramming experiment, we suggest to follow protocol reported in Box 2 to enable users to become familiar with microfluidic technology and to verify the chip functionality. In particular, we describe how to perform the coating of the culture surface and how to change medium. Following the explanations in Figure 3 and Supplementary Video 1, microfluidic culture can be performed efficiently, without bubble formation or evaporation of the medium.

Sequential RNA transfections (Steps 3-9). This part of the protocol includes daily transfections of mRNA mix for a period of 8 days. After 8 days the medium is changed to pluripotency medium for hiPSC colony stabilization and growth for another $\sim 7-9$ days. The RNA mix of the reprogramming kit used in this protocol includes three components ${ }^{7}$ : non-modified mRNA of the four Yamanaka factors (OCT4, SOX2, KLF4, C-MYC), plus NANOG, and LIN28; nonmodified mRNA encoding for $E 3, K 3$, and $B 18 R$ for evasion of the immune response and overcome RNA-mediated toxicity; and double-stranded microRNAs (miRNAs) from the 302/367 cluster. An increasing dose of the RNA mix is used in transfections from day 1 to day 8 to keep the transfected factor expression in the growing cell population high, while avoiding toxicity. The overall goal is to maintain approximately constant amounts of RNA transfected per cell.

In the section below "Quality control of main reagents", we give suggestions for checking that reagents are working and that cells are properly transfected. This could be useful also to investigate possible differences in performance of critical reagents as a consequence of batch variations.

Identification (Steps 11-15) and extraction of the hiPSC colonies (Step 27). After progressive morphological changes occurring during reprogramming, at day 14 the presence of TRA-1$60^{+}$hiPSC colonies can be verified by live staining, and the reprogramming efficiency quantified (Figure 4). Colonies need to grow for two more days to become larger before isolation.

Depending on the specific application, the colonies formed in a microfluidic chamber can be isolated as a pool or as mechanically picked single colonies (Figure 5 and Supplementary Video 2), and re-plated in standard wells for expansion ${ }^{9}$ and characterization ${ }^{20}$. The first method of passaging, based on ethylenediaminetetraacetic acid (EDTA), is less time consuming, but pools together all the colonies from a single culture chamber. Mechanical picking is more time demanding, and contaminates the hiPSCs with some fibroblasts (which can be removed at subsequent passages), but allows a single colony to expand. The success 
rate of re-plating the picked colonies depends on multiple factors, such as the size of cell clusters and the coating of the destination dish, as in standard hiPSC passaging.

\section{Level of expertise}

Microfluidic device production requires the use of photo- and soft-lithographic techniques, and requires the use of equipment not commonly available in a biological laboratory, but it can be easily outsourced. However, if the equipment is available, a graduate student can master these skills after 6 weeks of training (Box 1). Training in microfluidic cell culture, described in Box 2, requires one to three experiments to develop adequate competence, and does not necessitate prior knowledge of micro-scale technologies. To successfully perform the protocol of fibroblast reprogramming (Steps 1-27), a biological background with cell culture experience is sufficient. For characterization and passaging of the obtained hiPSC colonies,

following established methods that have been described previously ${ }^{9,20}$, pluripotent stem cell biology skills are required. 


\section{Box 1 | MICROFABRICATION}

The first three sub-steps shown in the workflow in Figure 2 refer to the stages required to fabricate the final microfluidic setup used in reprogramming experiments. The techniques involved are well-established in the field and we refer the reader to a published protocol for details on replica molding (REM) for fabrication of microfluidic devices in PDMS ${ }^{19}$. We provide the notes necessary to adapt the protocol to the specific device used in this work.

Reagents and equipment are listed within the list of materials for the main protocol.

\section{Procedure}

1| Photomask design (Step 1 in ref. 19 is only necessary if specific modifications to the layout proposed here are needed. The photomask for this protocol is provided as a highresolution supplementary manual. The layout is described in Figure S2.

2| Master mold fabrication (Step 4 and 6 in ref. 19) requires photolithography to fabricate a master with a $200-\mu \mathrm{m}$ high pattern, using negative SU-8 2100 resist. This mold can be used several times.

CRITICAL STEP Repeat Step 6 in ref. 19 every 10-15 uses of the mold for PDMS device production, to preserve it for long-term use.

3| To produce the microfluidic device (Steps 7-11 in ref. 19), use Sylgard 184 PDMS in a 10:1 base:curing-agent ratio in order to obtain PDMS stamps of roughly 3-mm thickness.

4| Use disposable 1- and 3-mm biopsy punches to produce each culture chamber inlet and outlet holes, respectively, as shown in Figure $1 \mathrm{~b}$.

5| Clean thoroughly a microscopy glass slide with MICRO-90 cleaning solution (2 vol- $\%$ in distilled water) and rinse with distilled water. Alternatively a rectangular coverslip glass can be used; in this case, cleaning is not needed.

6| Attach the patterned PDMS to a dry clean glass slide by an oxygen plasma cleaner (Step 34-36 in ref. 19).

7| Cool the bonded microfluidic device to room temperature $\left(25^{\circ} \mathrm{C}\right)$.

$8 \mid$ Pipette $20 \mu \mathrm{L}$ of isopropanol inside each culture chamber to clean the chamber from production process by-products, and rinse by flowing $30 \mu \mathrm{L}$ of distilled water into the chambers before isopropanol evaporation.

9| Dry the chambers by aspirating the water with a $200-\mu \mathrm{L}$ pipette and package the microfluidic device inside an autoclave bag.

10| Autoclave at $121^{\circ} \mathrm{C}$ for 15 minutes, and let it dry at the end.

PAUSE POINT The microfluidic devices can be kept in this state, at room temperature, indefinitely before use. 


\section{Box 2 | FIBROBLAST CULTURE IN MICROFLUIDICS}

Before using the microfluidic setup for cell reprogramming, it is useful to first test that the system allows fibroblast expansion. This test has two purposes: (i) become familiar with cell seeding and liquid handling in the microfluidic device; and (ii) confirm that there are no problems with the device. Reagents and equipment are listed within the list of materials of the main protocol.

\section{Procedure}

1| Prepare a vitronectin solution at $25 \mu \mathrm{g} / \mathrm{mL}$ in DPBS under sterile conditions and warm it up to room temperature. Prepare $12 \mu \mathrm{L}$ of solution per culture chamber, with an additional $10 \%$ excess volume. Alternatively, for fibroblast culture only, a fibronectin solution at $25 \mu \mathrm{g} / \mathrm{mL}$ in DPBS can be used.

CRITICAL STEP For long-term culture, the quality of glass surface coating is critical and high-quality recombinant proteins, such as vitronectin, reduce experimental variability.

2| Under laminar flow hood, extract a 10-chamber microfluidic chip from its sterile packaging using sterile tweezers, and place it inside a $100-\mathrm{mm}$ diameter Petri dish.

3| Pipette $12 \mu \mathrm{L}$ of vitronectin solution in each (empty) chamber, ensuring the tip is properly placed inside the 1-mm diameter inlet before injection (Figure 3b).

4| Add $1.5 \mathrm{~mL}$ of DPBS, optionally supplemented with penicillin/streptomycin, in the dish, around the microfluidic chip.

$5 \mid$ Incubate for 1 hour at room temperature.

6| Pipette $12 \mu \mathrm{L}$ of DMEM with 10\% FBS (or other fibroblast medium), optionally supplemented with penicillin/streptomycin, in each chamber, ensuring the tip is properly placed inside the 1-mm diameter inlet before injection (Figure 3b).

7| Aspirate the mixture of vitronectin solution and medium from the reservoir, avoiding complete emptying (Figure 3b). Check that no bubble is formed at the inlet after this procedure.

PAUSE POINT The chip can be left under the hood in this state for $\sim 1$ hour before cell seeding. Place at $+4^{\circ} \mathrm{C}$ for longer periods of time, preferably less than $\sim 12$ hours. Bring it back to room temperature before cell seeding.

8| Prepare a cell suspension by detaching the fibroblasts from the previous culture dish and resuspending them in DMEM with 10\% FBS (or other fibroblast medium), according to standard biological practice. To seed cells at $60 \mathrm{cell} / \mathrm{mm}^{2}$, a cell concentration in suspension of $300 \mathrm{cell} / \mu \mathrm{L}$ is required. Prepare $12 \mu \mathrm{L}$ of solution per culture chamber, with an additional $10 \%$ excess volume.

9| Pipette $12 \mu \mathrm{L}$ of well-mixed cell suspension in each chamber, ensuring the tip is properly placed inside the $1-\mathrm{mm}$ diameter inlet before injection. The injection should be fast to guarantee spatial cell homogeneity along the microfluidic chamber (Figure 3c).

10| Place the dish containing the seeded microfluidic chip in an incubator at $37^{\circ} \mathrm{C}$ and $5 \%$ $\mathrm{CO}_{2}$.

11| After 24 hours and approximately every 12 hours after that, replace the medium in the microfluidic chambers with pre-warmed fibroblast medium. For medium changes, deposit the $12-\mu \mathrm{L}$ droplet at the inlet without pipetting inside the chamber, repeat the 
operation for every chamber of the chip; last, with a p200 pipette aspirate the medium from each reservoir to let the medium enter the chambers by capillarity (Figure $3 \mathrm{~d}, \mathrm{f}$ and Supplementary Video 1).

CRITICAL STEP If medium changes every $12 \mathrm{~h}$ are not possible, avoid exceeding $15 \mathrm{~h}$ without medium changes.

12 Add $1 \mathrm{~mL}$ of sterile water, optionally supplemented with penicillin/streptomycin, in the dish, around the microfluidic chip, when the DPBS added at Step 4 dries out.

13| Observe by phase contrast microscopy the fibroblast growth during culture for a few days (Figure 3g). 


\section{MATERIALS}

\section{REAGENTS}

- Human fibroblasts (for example, BJ from ATCC, cat. \# CRL-2522 and HFF-1 from ATCC, cat. \# SCRC-1041)

CAUTION The cell lines used in your research should be regularly checked to ensure they are authentic and are not infected with mycoplasma.

- $\quad$ Trypsin- EDTA $0.25 \%$ (Gibco, cat. \# 25200056)

- Dulbecco's modified Eagles' medium (DMEM, high glucose; Gibco, cat. \# 11965)

- Fetal bovine serum (FBS; Gibco, cat. \# 10099141)

- Vitronectin (VTN-N; Gibco, cat. \# A14700)

- Fibronectin (Corning, cat. \# CB-40008)

- Dulbecco's phosphate-buffered saline (DPBS; Gibco, cat. \# 14190144)

- Sterile distilled water

- Penicillin/streptomycin (Gibco, cat. \# 15140122)

- SU-8 2100 negative resist (MicroChem)

- $\quad$ SYLGARD ${ }^{\mathrm{TM}} 184$ Silicone Elastomer Kit (Dow Corning)

CRITICAL SYLGARD ${ }^{\mathrm{TM}} 184$ Silicone Elastomer Kit enables long term cell culture; the use of other elastomeric silicones need to be proved.

- Microscopy glass slides (Menzel, cat. \# 10756991)

- Cover slips 24 x 60 mm (Menzel, cat. \# 15747592)

- StemMACS mRNA Transfection Kit (Miltenyi, cat. \# 130-104-463)

- eGFP mRNA (Stemgent, cat. \# 05-0020)

OPTIONAL eGFP mRNA can be used during the protocol to visualize the transfection efficiency.

- $\quad$ StemRNA-NM Reprogramming Kit (Stemgent, cat. \# 00-0076)

CRITICAL The use of other commercially available RNA-NM Reprogramming Kit or custom-made RNA have to be always optimized because they could have a strong effect on the reprogramming efficiency.

- RNaseZap RNase Decontamination Solution (Invitrogen, cat. \# AM9780)

- Pluriton medium (Stemgent, cat. \# 00-0070)

- Essential 6 Medium (E6; Gibco, cat. \# A1516401)

- Fibroblast growth factor 2 (FGF2; Peprotech, cat. \# 100-18B-1000)

- StemMACS iPS-Brew XF, human (Miltenyi Biotec, cat. \# 130-104-368)

- TeSR-E8 kit for hESC/hiPSC cell maintenance (E8; Stem Cell Technologies, cat. \# 05990)

- Rock Inhibitor StemMACS Y27632 (Miltenyi Biotec, cat. \# 130-103-922)

- LSD1inhibitor RN-1 (EMD Millipore, cat. \# 489479)

- Human iPS Reprogramming Boost Supplement II (EMD Millipore, cat. \# SCM094)

- StainAlive TRA-1-60 antibody (DyLight 488), mouse anti-human (Stemgent, cat. \# 090068) 
- Pierce 16\% formaldehyde (w/v), methanol-free (Thermo Fisher Scientific, cat. \# 28906) CAUTION It causes acute oral, dermal and inhalation toxicity. Handle it according to the manufacturer's instructions.

- Matrigel matrix growth factor reduced (GFR; Corning, cat. \# L003975)

- EDTA (0.5M), pH 8.0 (Thermo Fisher Scientific, cat. \# AM9260G)

- Horse serum (Thermo Fisher Scientific, cat. \# 16050122)

- Triton X-100 (Sigma, cat. \# T8787-100ML) CAUTION It is harmful if swallowed, causes skin irritation, serious eye damage, and is very toxic to aquatic life with long lasting effects. Handle it according to the manufacturer's instructions.

- Bovine serum albumin (Sigma, cat. \# A3912-100G)

- Mouse anti-human OCT4 antibody (SantaCruz, cat. \# sc-5279, 1:200 for immunofluorescence, 1:500 for Western blot)

- Rabbit anti-human SOX2 antibody (Novus Biological, cat. \# NB110- 37235, 1:200 for immunofluorescence, 1:500 for Western blot)

- Rabbit anti-human NANOG antibody (Reprocell, cat. \# RCAB004P- F, 1:200 for immunofluorescence)

- Rabbit anti-human NANOG antibody (Cell Signaling Techonolgy, cat. \#4903, 1:1000 for Western blot)

- Mouse anti-human TRA-1-60 antibody (Millipore, cat. \# MAB4360, 1:200 for immunofluorescence)

- Rabbit anti-human KLF4 polyclonal antibody (Santa Cruz, cat. \# sc-20691, 1:200 for immunofluorescence, 1:500 for Western blot)

- Anti GADPH (Cell Signaling Technology, cat. \# D16H11, 1:2000 for Western blot)

- Anti Vimentin (Genetex, cat. \# 100619, 1:2000 for Western blot)

- Goat anti-mouse $\operatorname{IgG}(\mathrm{H}+\mathrm{L})$ cross-adsorbed secondary antibody, Alexa Fluor 488 (Thermo Fisher Scientific, cat. \# A-11001)

- Goat anti-mouse $\operatorname{IgG}(\mathrm{H}+\mathrm{L})$ cross-adsorbed secondary antibody, Alexa Fluor 594 (Thermo Fisher Scientific, cat. \# A-11005)

- Goat anti-rabbit IgG (H+L) cross-adsorbed secondary antibody, Alexa Fluor 594 (Thermo Fisher Scientific, cat. \# A-11012)

- Goat anti-rabbit IgG $(\mathrm{H}+\mathrm{L})$ cross-adsorbed secondary antibody, Alexa Fluor 488 (Thermo Fisher Scientific, cat. \# A-11008)

- Hoechst 33342 (Thermo Fisher Scientific, cat. \# H3570)

\section{REAGENT SETUP}

\section{Preparation of the RNA mix for reprogramming}

Thaw the 3 vials of NM-RNAs provided in the StemRNA-NM Reprogramming Kit (OSKMNL NM-RNA, EKB NM-RNA, and NM-microRNAs) and prepare the RNA mix according to manufacturer's instructions. The daily RNA mix used for reprogramming a 10-chamber microfluidic chip ranges from 0.9 to $1.9 \mu \mathrm{L}$ (Table 1). We suggest preparing aliquots of $2 \mu \mathrm{L}$ in sterile RNase-free microcentrifuge tubes, in order to thaw a fresh aliquot every day. Store the aliquots at $-80^{\circ} \mathrm{C}$ for up to three months. During reprogramming, thaw the required vials on ice and do not refreeze. 
CRITICAL STEP First, use RNAse ZAP to clean all the surfaces of the laminar flow hood, use RNase-free sterile $0.2-\mathrm{mL}$ microcentrifuge tubes and RNAse-free sterile aerosol-barrier tips. Keep the RNA mix on ice at all times.

\section{Quality control of main reagents}

The quality control suggestions we describe here are optional and are useful to verify the quality of the main reagents. They can be performed either before starting the actual reprogramming experiment or for troubleshooting an unsuccessful experiment. In the first check, start a reprogramming experiment but stop approximately 24 hours after the fourth transfection. Verify robust expression of the encoded proteins by immunocytochemistry (Supplementary Figure 3a) and Western blot (Supplementary Figure 3b). Testing all six transcription factors is rarely needed, the pluripotency-related transcription factors (OCT4, NANOG, SOX2) should be given priority. At this point, cells should have already down-regulated the fibroblast marker Vimentin (VIM), which can also be assayed by Western blot (Supplementary Figure 3b). Due to variations in the transfectability of different cell lines, mRNA encoding green fluorescence protein (GFP) can be added to the transfection solution, and its induced expression verified by fluorescence microscopy starting 24 hours after the first transfection.

\section{EQUIPMENT}

- Laminar flow hood

- $\mathrm{CO}_{2}$ water-jacketed incubator, set at $37^{\circ} \mathrm{C}, 5 \% \mathrm{CO}_{2}$, and $5 \% \mathrm{O}_{2}$

- Thermostatic water bath set at $37^{\circ} \mathrm{C}$

- Phase contrast and epifluorescence microscope

- Stereomicroscope

- Sterile tweezers

- Sterile scalpel

- Disposable borosilicate glass Pasteur pipettes

- DNase and RNase-free 0.2- and 1.5-mL sterile microcentrifuge tubes

- RNase-free sterile $15-\mathrm{mL}$ conical tubes

- RNase-free sterile 50-mL conical tubes

- RNase-free sterile aerosol-barrier tips (10, 20, 200 and 1,000 $\mu \mathrm{L})$

- Polystyrene tissue culture-treated six-well plates (BD Falcon, cat. \# 353046)

- Polystyrene tissue culture-treated 24-well plates (BD Falcon, cat. \# 353047)

- $100-\mathrm{mm}$ diameter Petri dish

\section{PROCEDURE}

\section{Cell seeding and RNA transfections TIMING $\sim 9 \mathrm{~d}$}

1| Prepare the 10-chamber microfluidic devices according to Box 1.

2| Day 0, at 2 PM. Repeat Steps 1-10 of Box 2 to seed human fibroblasts to be reprogrammed in microfluidics, and place them in incubator set to hypoxic conditions $\left(37^{\circ} \mathrm{C}, 5 \% \mathrm{CO}_{2}, 5 \% \mathrm{O}_{2}\right)$ overnight. The incubator used should be set to hypoxic conditions for the whole duration of the experiment. 
CRITICAL STEP In parallel, we recommend seeding a few channels with fibroblasts that will not be transfected, as a control for any morphological changes that occur during reprogramming.

3| Day 1, 9 a.m. ( 16 hours later). Replace medium with $12 \mu \mathrm{L}$ of supplemented Pluriton medium per chamber (Figure 3d, f and Supplementary Video 1). Alternatively, E7 medium (E6 supplemented with FGF2 at $100 \mathrm{ng} / \mathrm{mL}$ ) can be used in place of supplemented Pluriton medium. The medium can be supplemented with penicillin/streptomycin, but this is optional.

CRITICAL STEP Cells should be put in reprogramming medium for at least 9 hours before transfection.

CRITICAL STEP Cells should have reached $\sim 50-60 \%$ confluence before the first transfection.

\section{? TROUBLESHOOTING}

4| Day 1, 6 p.m. ( $\sim 9$ hours later). Prepare a low-dose transfection solution at room temperature, using the StemMACS mRNA transfection kit, keeping RNA on ice. Specifically, prepare separately Solution 1 and Solution 2, according to Table 1, and gently mix each of them. Put Solution 2 in Solution 1 and mix by gently pipetting 4 times.

CRITICAL STEP Use RNAse-free tubes and tips.

CRITICAL STEP Use a freshly thawed aliquot of RNA every day.

$5 \mid$ Incubate the mixture at room temperature for 20 minutes.

6| Add the transfection solution (mixture of Solution 1 and Solution 2) to Solution 3 (supplemented Pluriton medium), pre-warmed to room temperature, and gently mix. Alternatively, E7 medium can be used in place of supplemented Pluriton medium. The medium can be optionally supplemented with penicillin/streptomycin.

7| Take cells out of the incubator, empty the reservoirs of the microfluidic device, and gently pipette $12 \mu \mathrm{L}$ of transfection solution inside each chamber (Figure 3e).

CRITICAL STEP Steps 6-7 should take less than $15 \mathrm{~min}$. Consider preparing the transfection mix multiple times if a high number of chips is reprogrammed.

8| Incubate cells overnight at $37^{\circ} \mathrm{C}$, in the hypoxic incubator.

9| On days 2-8, repeat Steps 3-8 with the following RNA doses according to Table 1: Day 2, low dose; Days 3-5, medium dose; and Days 6-8, high dose. On days 1-8, medium can be supplemented with LSD1 inhibitor at a final concentration of $1 \mu \mathrm{M}$, to further promote mesenchymal-to-epithelial transition. On Days 5-8, medium can be supplemented with Human iPS Reprogramming Boost Supplement II (containing sodium butyrate, PS48 and TGF- $\beta$ RI Kinase Inhibitor IV), to further enhance reprogramming efficiency.

\section{? TROUBLESHOOTING}

\section{hiPSC colony identification and growth TIMING $\sim 7 \mathrm{~d}$}

10| On Days 9-15, replace medium in the morning ( $\sim 9$ a.m.) and at night ( $\sim 7$ p.m.) with 12 $\mu \mathrm{L}$ per chamber of hiPSC expansion medium, e.g. StemMACS iPSBREW XF or E8 medium. 
11| When hiPSC colonies start to be visible ( Days 10-14), verify the expression of the pluripotency marker TRA-1-60 by live staining. To do this, first prepare a solution of Stemgent StainAlive TRA-1-60 antibody at $5 \mu \mathrm{g} / \mathrm{mL}$ and inject $12 \mu \mathrm{L}$ per chamber.

12| Incubate the cells for $30 \mathrm{~min}$ in the incubator.

13| Inject $12 \mu \mathrm{L}$ of medium per chamber and incubate for 5 minutes.

14| Repeat Step 13, twice.

15| Observe the culture chambers by fluorescence microscopy (EX/EM: 488/525) (typical results can be seen in Figure 4b).

16| Continue culture as described in Step 10.

\section{Immunostaining for pluripotency markers in microfluidics TIMING $\sim 2 \mathrm{~d}$}

17| After hiPSC colony identification, some chambers can be used for hiPSC immunofluorescence characterization. Use $12 \mu \mathrm{L}$ volume per chamber for each of the following steps. First wash the chambers once with DPBS and then incubate the cells with $4 \%$ formaldehyde for 10 minutes at room temperature.

$18 \mid$ Wash three times for 5 minutes with DPBS.

19| Incubate cells with blocking solution (DPBS with 10\% HS, add $0.1 \%$ Triton for intracellular targets) for 45 minutes at room temperature.

20| Prepare final-concentration primary antibody solutions, inject into the microfluidic chambers, and incubate overnight at $+4^{\circ} \mathrm{C}$. We recommend using the following primary antibodies:

21| Wash three times for 5 minutes with DPBS.

22| Inject secondary antibody solution and incubate for 2 hours at room temperature. The following secondary antibodies are needed if using the primary antibodies recommended in step 20:

23| Wash with DPBS for 5 minutes.

24| Incubate with $1 \mu \mathrm{g} / \mathrm{ml}$ of HOECHST in DPBS for 10 minutes at room temperature.

25 | Wash with DPBS.

26| Image cells using an epifluorescence microscope.

\section{hiPSC colony extraction}

27| Extract colonies by EDTA (option A) or manual picking (option B).

\section{Option A) hiPSC colony extraction by EDTA TIMING $\sim \mathbf{4}$ h}

i. Prepare a six-well plate coated with Matrigel Reduced Factor (MRF) 0.5\%, incubating for 1 hour at room temperature. Alternatively, plates can be coated with vitronectin (VTN-N) according to manufacturer's instructions.

CRITICAL STEP The MRF-coated plate can be prepared in advance (up to 2 weeks). In this case, pre-warm at $37^{\circ} \mathrm{C}$ for 30 minutes before use.

ii. Supplement the hiPSC expansion medium with $1 \mu \mathrm{L} / \mathrm{mL}$ of Rock Inhibitor (RI).

iii. Aspirate the MRF coating from the plates, avoiding drying, and replace with the medium prepared in Step 27ii.

iv. Wash the microfluidic chamber three times with $12 \mu \mathrm{L}$ of calcium- and magnesium-free DPBS.

v. Wash the microfluidic chamber five times with $12 \mu \mathrm{L}$ of $0.5 \mathrm{mM}$ EDTA. 
vi. Incubate $5-10$ minutes at $37^{\circ} \mathrm{C}$ until hiPSC colonies have a less compact morphology (Figure 5a).

CRITICAL STEP If hiPSC colonies are detached proceed to Step vii, otherwise incubate for additional 5 minutes.

vii. Remove excess solution from the reservoir.

viii. Pipette in and out of the chamber $12 \mu \mathrm{L}$ of the medium prepared in Step 27ii.

ix. Transfer the $12 \mu \mathrm{L}$ of medium from Step 27viii into the MRF-coated well plate.

$\mathrm{x}$. Under the microscope check the successful transfer of the clone, if the hiPSC clone/clones is still present in the chamber repeat steps vii-viii.

xi. Completely dry the microfluidic chambers, transferring the cell suspension medium into the MRF-coated well plate.

xii. Replace medium in the well after 48 hours, with hiPSC expansion medium without RI.

xiii. Expand and characterize the hiPSC clones as previously described ${ }^{9,20}$ or according to the manufacturer's instructions for the products used.

\section{Option B) hiPSC single colony extraction by mechanical picking TIMING $\sim 1$ d}

i. Prepare some Pasteur pipettes with the tips curved using a Bunsen burner, and autoclave them.

ii. Perform Steps 27 i-vii using a 24-well plate.

iii. Use a stereomicroscope to identify the hiPSC colony to pick.

iv. In sterile conditions, under a stereomicroscope, use a scalpel to cut the microfluidic chamber as described in Figure 5b and Supplementary Video 2.

CRITICAL During the first two cuts (Figure 5b), make sure to cut slightly outside the chamber to avoid pushing the ceiling of the chamber against the hiPSC colony.

v. Use sterile tweezers to remove the chamber ceiling (Figure 5b).

vi. Add some medium on the open microfluidic culture chamber to avoid cell drying and improve optical observation.

vii. Under the stereomicroscope, use a glass Pasteur pipette prepared in Step 31 to mechanically scratch the colony from the surface and transfer it into the plate prepared in Step 32.

viii. Repeat Steps iii-vii for next colonies.

CRITICAL Avoid prolonged exposure of the cells to room temperature. Place the microfluidic system back in the incubator for 30 minutes between operations in different chambers.

\section{? TROUBLESHOOTING}

See Table 2 for troubleshooting details.

\section{TIMING}

Step 1, preparation of microfluidic devices: $\sim 4 \mathrm{~h}$.

Step 2, Cell seeding $\sim 3 \mathrm{~h}$, performed once, at the beginning of reprogramming. Step 3, Medium change $\sim 30$ min, for one chip every morning ( $\sim 9$ AM) Day 1-8. Steps 4-9, RNA transfections $\sim 1 \mathrm{~h}$, for one chip every night ( $\sim 6$ PM) Day 1-8. Step 10, Medium change $\sim 30$ min (twice per day, $\sim 9$ AM and $\sim 6$ PM) Day 9-15. Steps 11-16, TRA-1-60 staining and fluorescence microscope observation $\sim 4 \mathrm{~h}$. 
Step 17-26, Immunostaining for pluripotency markers in microfluidics, $\sim 2 \mathrm{~d}$. The overall work load for immunofluorescence assay in microfluidics is similar to that in standard wells.

Steps 27 Option A, hiPSC colony extraction by EDTA, $\sim 4 \mathrm{~h}$

Steps 27 Option B , hiPSC single colony extraction by mechanical picking, $\sim 1 \mathrm{~d}$

Box 1, step 1, (optional) photomask design: $\sim 2 \mathrm{~h}$, with prior knowledge of the software used

Box 1, step 2, master mold fabrication: $5 \mathrm{~h}$

Box 1, step 3, microfluidic device production: $3 \mathrm{~h}$

Box 1, steps 4-10, microfluidic device assembly and sterilization: $1 \mathrm{~h}$

Box 2, steps 1-10, cell-seeding protocol: $3 \mathrm{~h}$

Box 2, step 11, medium changes for one chip: 10-30 min, twice per day

Box 2, steps 12 and 13, quality control of fibroblast culture: 10-20 min

\begin{abstract}
ANTICIPATED RESULTS
It is advisable to first set up the process using, as a standard, low-passage human foreskinderived fibroblasts, such as BJ or HFF-1. This protocol is effective in obtaining hiPSC colonies in every culture chamber. The timing of the morphological changes of cells along reprogramming, from acquisition of an epithelial morphology to compaction of hiPSC colonies, are shown in Figure 4. By days 3-4, cells start to change morphology towards an epithelial-like state, which is acquired by day 7. Between days 8-10, small clusters of epithelial cells start to emerge. Defined cell nuclei are visible with evident nucleoli. On day 14, we found BJ reprogramming efficiency was $52 \pm 5$ TRA- $1-60^{+}$colonies per chamber (mean \pm s.d., $\mathrm{n}=60$ ), as shown in Figure 4c and quantified in Figure 4d (see also Supplementary Data 1).

The reprogramming efficiency can be further increased by adding previously reported reprogramming boosters. As proof of concept we report the use of LSD1 inhibitor ${ }^{21}$ (efficiency $70 \pm 5$, mean \pm s.d., $n=24$ ) or a cocktail of sodium butyrate ${ }^{22}$, PS $48^{23}$, and TGF- $\beta$ RI Kinase Inhibitor $\mathrm{IV}^{24}$ (efficiency 160 \pm 20 , mean \pm s.d., $\mathrm{n}=48$ ) (Figure 4d and Supplementary Data 1).

Reprogramming in E6 supplemented with FGF2 (100 ng/mL), without adding other reprogramming boosters, shows lower efficiency compared to Pluriton medium: BJ efficiency is $19 \pm 4$ TRA- $1-60^{+}$colonies per chamber (mean \pm s.d., $n=5$ ), and HFF-1 efficiency is $27 \pm 7$ TRA-1$60^{+}$colonies per chamber (mean \pm s.d., $n=10$ ), both by day 14 (Figure $4 d$ and Supplementary Data 1). To improve reprogramming efficiency, if high proliferation rate is observed in E7 medium, cells can be seeded at lower density $(30$ cell $/ \mathrm{mm} 2)$, whereas, in case transfections induce high toxicity, they can be kept at low dose until cells reach $\sim 60 \%$ confluence, Typically, the transfection is switched to medium dose at day 5-6 for BJ or HFF-1 fibroblasts.
\end{abstract}

From our experience, the reprogramming efficiency is very variable from cell line to cell line ${ }^{2}$. In the troubleshooting section we give some suggestions on how to handle this, by changing various factors, such as cell density, RNA transfection dose, and number of transfections. Given the high throughput and low cost of microfluidics, for new primary lines it is recommended to test multiple reprogramming conditions in parallel, according to the principles described in the troubleshooting section, in 1-2 microfluidic devices. Following this strategy $\sim 30 \%$ of the microfluidic chambers contain up to 5 colonies, which is sufficient for derivation of the few stable hiPSC lines usually required for use. Despite some flexibility being required to identify 
the optimal conditions, we consistently found a gain in efficiency when performing the process in microfluidics compared to using standard well plates.

\section{ACKNOWLEDGEMENTS}

This work was supported by Natural Science Foundation of China (No. 31601178), ShanghaiTech University, University of Padova (TRANSAC and PRAT), CaRiPaRo Foundation, Telethon Foundation (GGP15275), and Oak Foundation Award (No. W1095/OCAY-14-191).

\section{AUTHOR CONTRIBUTIONS}

O.G., C.L., and N.E. designed the study and wrote the manuscript. O.G. and C.L. optimized and performed the reprogramming experiments. W.Q. and S.G. produced the microfluidic devices. W.Q., E.B., and E.T. helped in reprogramming experiments. E.B., E.T. and A.U. helped in cell characterization. N.E. oversaw the project.

\section{COMPETING INTEREST STATEMENT}

O.G, C.L. and N.E. are coinventors on patent applications describing the reprogramming and differentiation processes in microfluidics, application number PD2013A000220, IT UA20162645 and 102016000039189 and PCT/IB2017/052167. O.G. and N.E. are co-founders of uSTEM Srl and Onyel Biotech Srl.

\section{DATA AVAILABILITY}

The authors declare that the main data supporting the findings of this study are available within the article and its Supplementary Information files.

\section{SUPPLEMENTARY INFORMATION}

- Figure S1 shows the results of fibroblast reprogramming using Simplicon RNA Reprogramming Technology;

- Figure S2 shows the design of the photomask used for producing the microfluidic device;

- Figure S3 shows the expected results of the quality control of the main reagents;

- Figure S4 shows conditions where some troubleshooting is needed;

- Supplementary Data 1 reports the numerical data used for producing Figure 4d;

- Supplementary Manual. High-resolution photomask for the microfluidic device production.

- Video S1 shows how to change medium in the microfluidic chambers;

- Video S2 shows how to perform hiPSC colony mechanical picking. 


\section{REFERENCES}

1. Takahashi, K. et al. Induction of Pluripotent Stem Cells from Adult Human Fibroblasts by Defined Factors. Cell 131, 861-872 (2007).

2. Luni, C. et al. High-efficiency cellular reprogramming with microfluidics. Nat. Methods 13, 446-452 (2016).

3. Banito, A. et al. Senescence impairs successful reprogramming to pluripotent stem cells Senescence impairs successful reprogramming to pluripotent stem cells. 2134-2139 (2009). doi:10.1101/gad.1811609

4. Luni, C., Serena, E. \& Elvassore, N. Human-on-chip for therapy development and fundamental science. Curr. Opin. Biotechnol. 25, 45-50 (2014).

5. Giobbe, G. G. et al. Functional differentiation of human pluripotent stem cells on a chip. Nat. Methods 12, 1-7 (2015).

6. Gagliano, O., Elvassore, N. \& Luni, C. Microfluidic technology enhances the potential of human pluripotent stem cells. Biochem. Biophys. Res. Commun. 473, 683-687 (2016).

7. Poleganov, M. A. et al. Efficient Reprogramming of Human Fibroblasts and BloodDerived Endothelial Progenitor Cells Using Nonmodified RNA for Reprogramming and Immune Evasion. Hum. Gene Ther. 26, 751-766 (2015).

8. Yoshida, Y., Takahashi, K., Okita, K., Ichisaka, T. \& Yamanaka, S. Hypoxia Enhances the Generation of Induced Pluripotent Stem Cells. Cell Stem Cell 5, 237-241 (2009).

9. Beers, J. et al. Passaging and colony expansion of human pluripotent stem cells by enzyme-free dissociation in chemically defined culture conditions. Nat. Protoc. 7, 2029 2040 (2012).

10. FUSAKI, N., BAN, H., NISHIYAMA, A., SAEKI, K. \& HASEGAWA, M. Efficient induction of transgene-free human pluripotent stem cells using a vector based on Sendai virus, an RNA virus that does not integrate into the host genome. Proc. Japan Acad. Ser. $B$ 85, 348-362 (2009).

11. Schlaeger, T. M. et al. A comparison of non-integrating reprogramming methods. Nat. Biotechnol. 33, 58-63 (2015).

12. Churko, J. M. et al. Transcriptomic and epigenomic differences in human induced pluripotent stem cells generated from six reprogramming methods. Nat. Biomed. Eng. 1, 826-837 (2017).

13. Okita, K. et al. A more efficient method to generate integration-free human iPS cells. Nat. Methods 8, 409-412 (2011).

14. Yoshioka, N. et al. Efficient generation of human iPSCs by a synthetic self-replicative RNA. Cell Stem Cell 13, 246-254 (2013).

15. Warren, L. et al. Highly efficient reprogramming to pluripotency and directed differentiation of human cells with synthetic modified mRNA. Cell Stem Cell 7, 618-630 (2010).

16. Mandal, P. K. \& Rossi, D. J. Reprogramming human fibroblasts to pluripotency using modified mRNA. Nat. Protoc. 8, 568-582 (2013).

17. Kogut, I. et al. High-efficiency RNA-based reprogramming of human primary fibroblasts. Nat. Commun. 9, (2018).

18. Melin, J. \& Quake, S. R. Microfluidic Large-Scale Integration: The Evolution of Design Rules for Biological Automation. Annu. Rev. Biophys. Biomol. Struct. 36, 213-231 (2007). 
19. Qin, D., Xia, Y. \& Whitesides, G. M. Soft lithography for micro- and nanoscale patterning. Nat. Protoc. 5, 491-502 (2010).

20. Martí, M. et al. Characterization of pluripotent stem cells. Nat. Protoc. 8, 223-253 (2013).

21. Cacchiarelli, D. et al. Integrative Analyses of Human Reprogramming Reveal Dynamic Nature of Induced Pluripotency. Cell 162, 412-424 (2015).

22. Mali, P. et al. Butyrate Greatly Enhances Derivation of Human Induced Pluripotent Stem Cells by Promoting Epigenetic Remodeling and the Expression of PluripotencyAssociated Genes. Stem Cells 28, 713-720 (2010).

23. Zhu, S. et al. Reprogramming of human primary somatic cells by OCT4 and chemical compounds. Cell Stem Cell 7, 651-655 (2010).

24. Ichida, J. K. et al. A Small-Molecule Inhibitor of Tgf- $\beta$ Signaling Replaces Sox2 in Reprogramming by Inducing Nanog. Cell Stem Cell 5, 491-503 (2009). 
TABLE 1 | Composition of transfection solutions for a 10-chamber microfluidic chip. (TB: transfection buffer; TR: transfection reagent)

\begin{tabular}{|c|c|c|}
\hline & \multicolumn{3}{|c|}{ Low dose } \\
\hline Solution 1 & Solution 2 & Solution 3 \\
\hline RNA mix: $0.9 \mu \mathrm{L}$ & TR: $0.3 \mu \mathrm{L}$ & Medium: $110.7 \mu \mathrm{L}$ \\
\hline TB: $3.7 \mu \mathrm{L}$ & TB: $4.4 \mu \mathrm{L}$ & \\
\hline \multicolumn{3}{|c|}{ Medium dose } \\
\hline Solution 1 & Solution 2 & Solution 3 \\
\hline RNA mix: $1.6 \mu \mathrm{L}$ & TR: $0.47 \mu \mathrm{L}$ & Medium: $104.4 \mu \mathrm{L}$ \\
\hline TB: $6.3 \mu \mathrm{L}$ & TB: $7.3 \mu \mathrm{L}$ & \\
\hline \multicolumn{3}{|c|}{ High dose } \\
\hline Solution 1 & Solution 2 & Solution 3 \\
\hline RNA mix: $1.9 \mu \mathrm{L}$ & TR: $0.6 \mu \mathrm{L}$ & Medium: $101.3 \mu \mathrm{L}$ \\
\hline TB: $7.5 \mu \mathrm{L}$ & TB: $8.8 \mu \mathrm{L}$ & \\
\hline
\end{tabular}


TABLE 2 | Troubleshooting table.

\begin{tabular}{|c|c|c|c|}
\hline STEP & PROBLEM & \begin{tabular}{|l} 
POSSIBLE REASON \\
\end{tabular} & SOLUTION \\
\hline Step 3 & $\begin{array}{l}\text { Before the first } \\
\text { transfection, cells did } \\
\text { not reach } 50-60 \% \\
\text { confluence. }\end{array}$ & $\begin{array}{l}\text { - Cells have reduced } \\
\text { proliferative ability. } \\
\text { - Cells did not attach } \\
\text { well during seeding } \\
\text { due to low-quality } \\
\text { coating or high } \\
\text { mortality. }\end{array}$ & $\begin{array}{l}\text { - } \text { Repeat cell seeding } \\
\text { with higher cell } \\
\text { density } \quad(70-80 \\
\left.\text { cell/mm }{ }^{2}\right) \text {. } \\
\text { - Change medium on } \\
\text { Day } 1 \text { at } \sim 9 \text { AM and } \\
\sim 7 \text { PM, and } \\
\text { continue on Day } 2 \\
\text { form Step 3. It will } \\
\text { reduce } \\
\text { reprogramming } \\
\text { efficiency, but } \\
\text { hiPSC colonies can } \\
\text { still be obtained. }\end{array}$ \\
\hline Step 9 & $\begin{array}{l}\text { Cell number is not } \\
\text { increasing during the } \\
\text { first days of } \\
\text { transfection. }\end{array}$ & $\begin{array}{l}\text { - Cells have reduced } \\
\text { proliferative ability. } \\
\text { - Cells are sensitive to } \\
\text { transfection toxicity. }\end{array}$ & $\begin{array}{l}\text { - Keep } \\
\text { transfection to low } \\
\text { dose. } \\
\text { - Consider seeding } \\
\text { more cells (70-80 } \\
\left.\text { cell } / \mathrm{mm}^{2}\right) \text {, if } \\
\text { reprogramming of } \\
\text { this cell line is } \\
\text { repeated. }\end{array}$ \\
\hline Step 9 & $\begin{array}{l}\text { Cells are almost } \\
\text { confluent at Day } 3 \\
\text { (Supplementary } \\
\text { Figure 4a). }\end{array}$ & $\begin{array}{l}\text { - Cells have high } \\
\text { proliferative ability. }\end{array}$ & $\begin{array}{l}\text { - Increase RNA dose } \\
\text { to high dose. } \\
\text { - Consider seeding } \\
\text { less cells (40-50 } \\
\left.\text { cell//mm }{ }^{2}\right), \\
\text { reprogramming of } \\
\text { this cell line is } \\
\text { repeated. }\end{array}$ \\
\hline Step 9 & $\begin{array}{l}\text { Cell morphology does } \\
\text { not show } \\
\text { mesenchymal-to- } \\
\text { epithelial transition at } \\
\text { Day } 5 \text { (Supplementary } \\
\text { Figure 4b). }\end{array}$ & $\begin{array}{l}\text { - Low transfectability } \\
\text { of cells. }\end{array}$ & $\begin{array}{l}\text { - Verify expression of } \\
\text { transcription factors } \\
\text { by } \\
\text { immunofluorescenc } \\
\text { e assay. } \\
\text { - Repeat the process } \\
\text { with higher dose }\end{array}$ \\
\hline
\end{tabular}




\begin{tabular}{|l|l|l|l|}
\hline Step 9 & & & \multicolumn{2}{|c|}{ RNA transfection. } \\
& $\begin{array}{l}\text { Cells with epithelial } \\
\text { morphology are not } \\
\text { making clusters at } \\
\text { Day 8. }\end{array}$ & $\begin{array}{l}\text { Some cell lines take } \\
\text { more time to Consider increasing } \\
\text { reprogram. } \\
\text { the number of } \\
\text { transfections. Use } \\
\text { BJ or HFF-1 } \\
\text { morphology at Day } \\
8 \text { as a reference for } \\
\text { deciding when to } \\
\text { stop the } \\
\text { transfections. }\end{array}$ \\
\hline
\end{tabular}




\section{Figures}

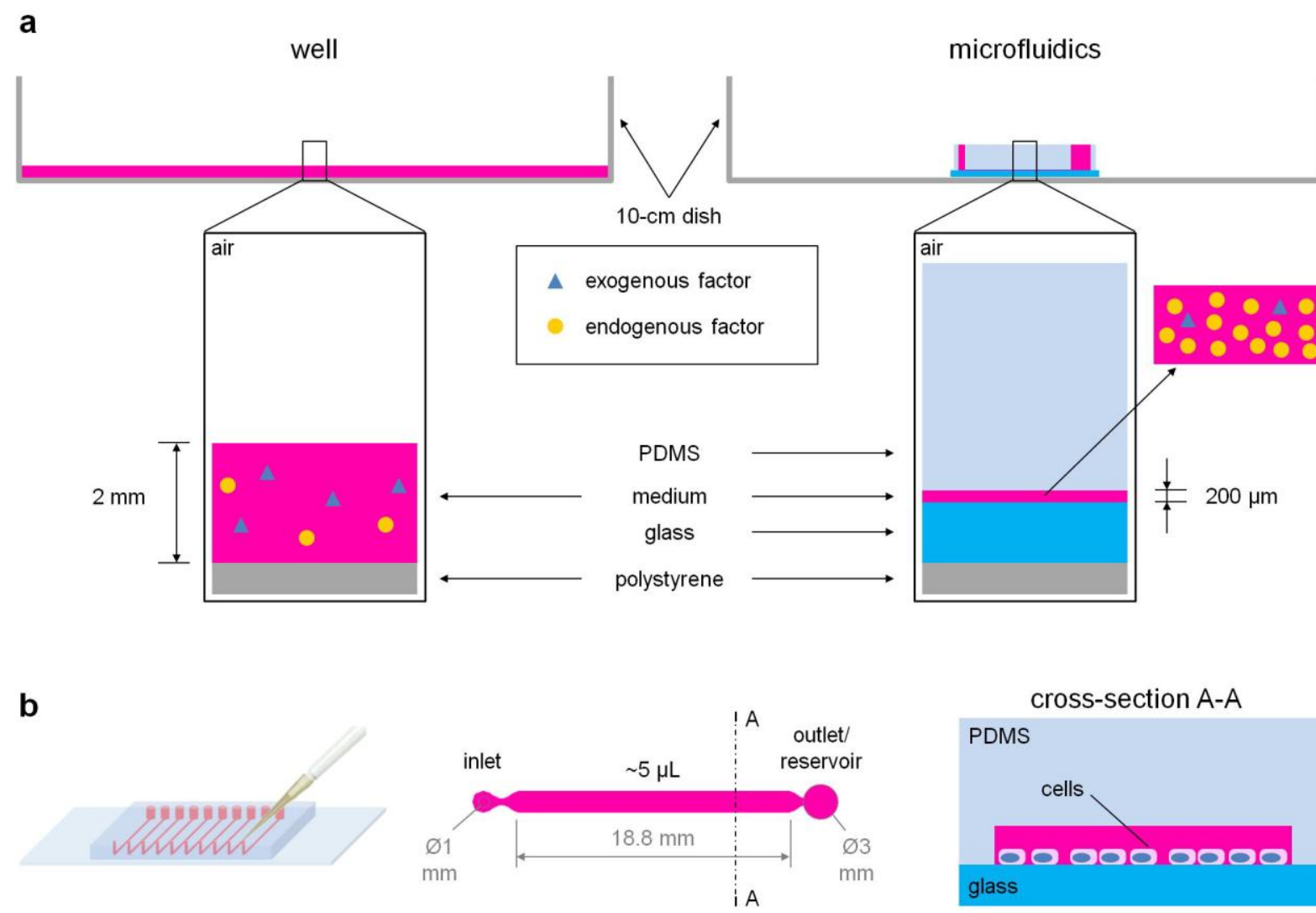

Figure 1 | Microfluidics down-scale and setup. (a) Schematic representation of medium reduction and endogenous factor accumulation from a standard well culture system to microfluidics. Endogenous and exogenous factor sizes are not to scale. (b) Drawing of a 10chamber microfluidic device during medium change by manual pipetting, and dimensions of a single channel-shaped microfluidic culture chamber. Cell size is not on scale. 


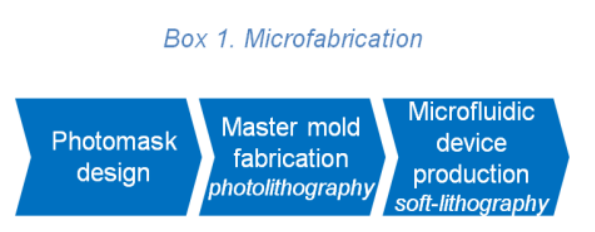
Box 2. Fibroblast culture
in microfluidics

Cell culture
surface
coating
Fibroblast
seeding

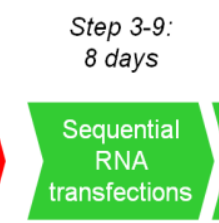
Step 11-15:
4 hours

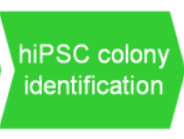

\begin{tabular}{c} 
Pooled hiPSC \\
colony isolation \\
(EDTA) \\
\hline $\begin{array}{c}\text { Single hiPSC } \\
\text { colony picking } \\
\text { (mechanical) }\end{array}$ \\
\hline
\end{tabular}

Step 27 B: 1 day

Figure 2 | Main stages of the protocol. 


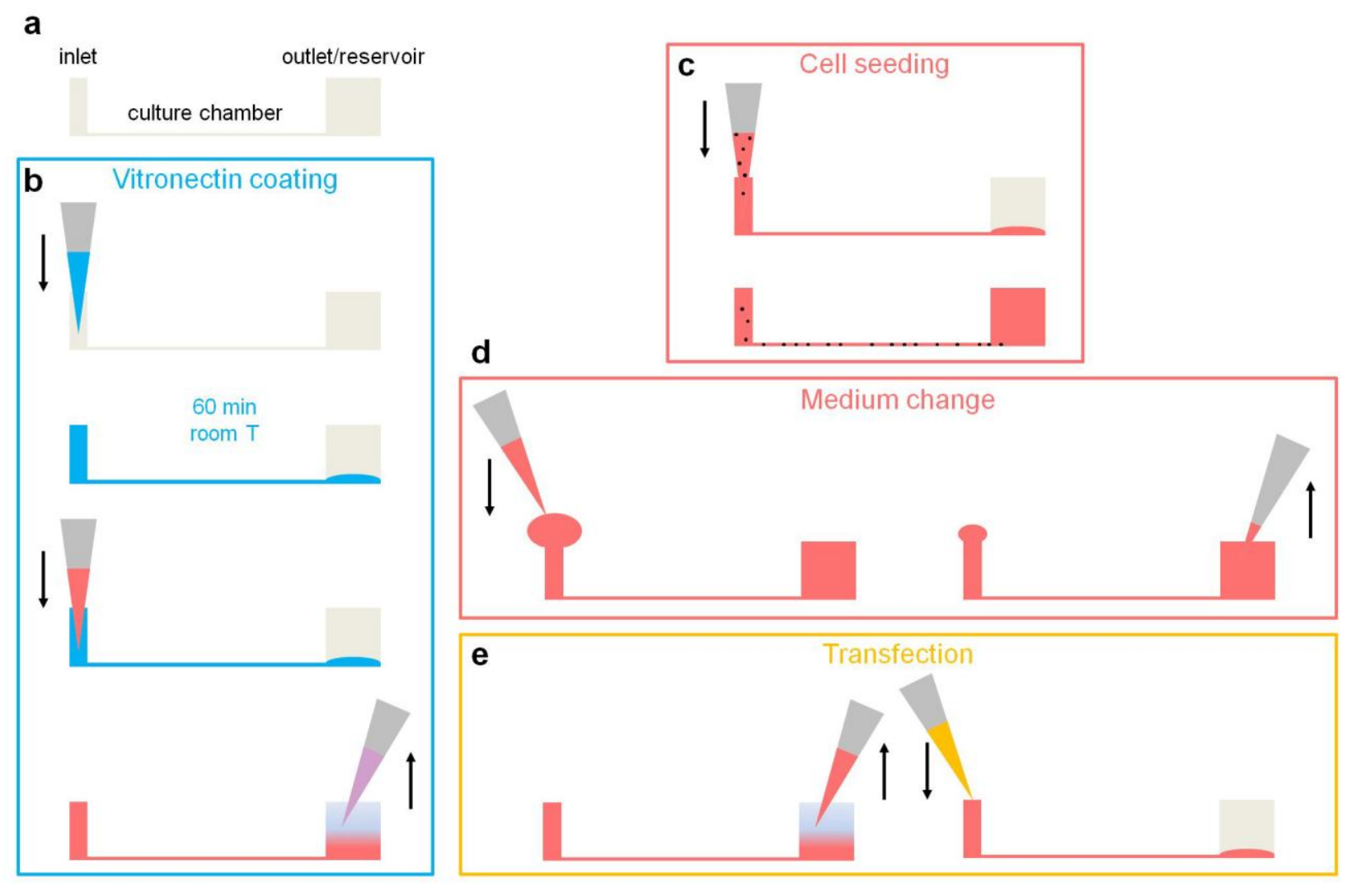

f
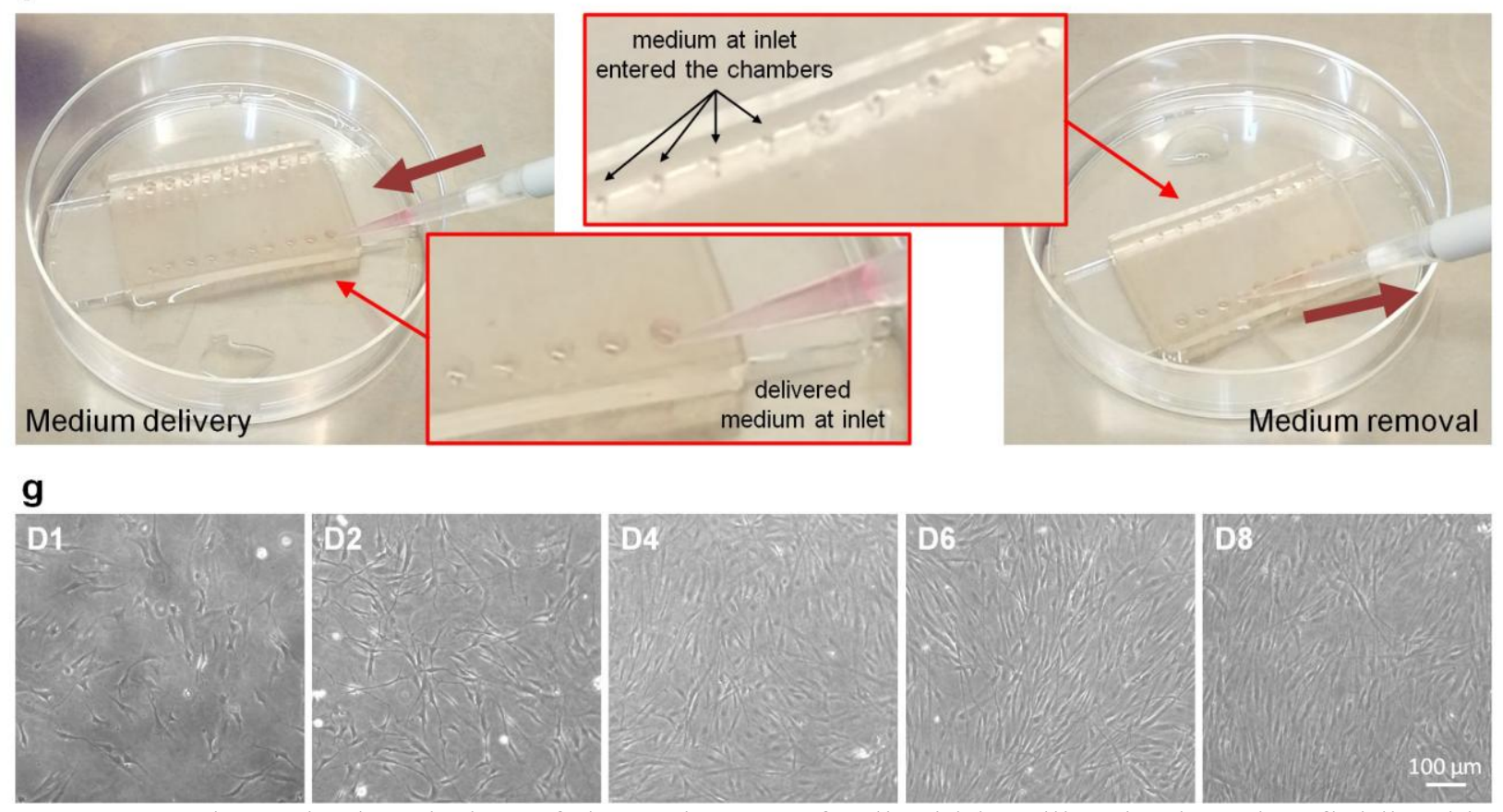

Figure 3 | Schematic description of the main steps for liquid handling in the microfluidic chip and images of the cell morphology that should be present. (a) A longitudinal vertical section of a microfluidic chamber is shown highlighting the different parts. (b) Vitronectin coating is performed when the culture chamber is still empty. To ease the inflow of protein solution, the tip should go precisely inside the inlet hole. A volume of $12 \mu \mathrm{L}$ is sufficient to fill the chamber and 
avoid bubble formation in the chamber due to evaporation during the 60 -minute incubation at room temperature. At the end of the incubation period, the protein solution is replaced with fresh medium that is left in the chamber until cell seeding, usually within an hour. Part of the solution in the reservoir, containing a mixture of Vitronectin solution and medium is aspirated, without completely emptying the reservoir. (c) Also during cell seeding, the tip should be placed precisely inside the inlet hole. $12-\mu \mathrm{L}$ of well-mixed cell suspension should be injected by quick pipetting. The reservoir will be full at the end of seeding and it is possible a little amount of medium is left at the inlet; it is convenient not to aspirate this medium, to obtain a spatially homogeneous seeding along the culture chamber. (d) During medium changes in the next days, the strategy that minimizes bubble formation and time consists in injecting $12 \mu \mathrm{L}$ of medium near the inlet; because the reservoir is almost full, a droplet of medium is formed at the inlet. Repeat this operation for all the chambers of the chip. Then, using a $200-\mu \mathrm{L}$ pipette, aspirate from each reservoir the amount of medium that is necessary to have all the medium at the inlet entering the chamber (it enters by capillarity). (e) Unlike during medium change, during transfection it is extremely important that the transfection solution goes completely inside the channel immediately to avoid inhomogeneities of cell transfection and toxicity. Thus, the reservoir should be emptied first, almost completely, and the transfection solution should be then injected approximately near the inlet. Letting capillarity making the solution entering the channel guarantees an approximately constant flow rate during successive medium changes and transfections. (f) Images of two stages during medium change: $12-\mu \mathrm{L}$ medium delivery at the inlet of the culture chamber, medium removal at the outlet/reservoir. Note that during medium removal the microfluidic chip is rotated of $180^{\circ}$. The two respective insets highlight the inlets of the channel after medium delivery and after medium entrance into the channel by capillarity. See also Supplementary Video 1. (g) Time course of fibroblast morphology during proliferation within a microfluidic chamber, at day 1, 2, 4, 6, 8 after seeding at $60 \mathrm{cell} / \mathrm{mm}^{2}$. 

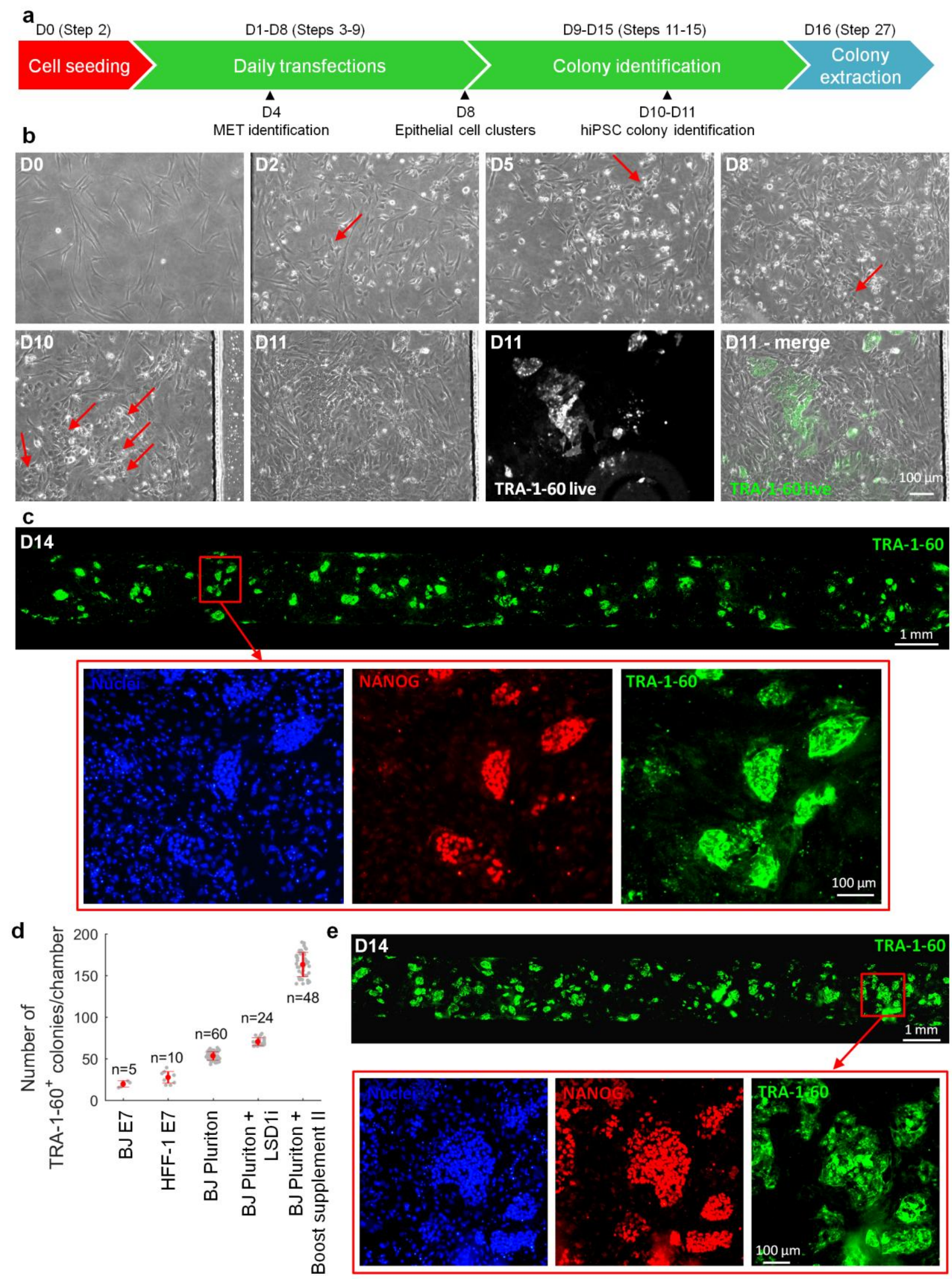
Figure 4 | Expected results during the human fibroblast reprogramming process. (a) Diagram of the work flow with indicated the critical morphological changes. (b) Time lapse of cell morphology along the process. Red arrows highlight morphological hallmarks: epithelial morphology (D2), cell clusters at different compacting stages (D5 and D8), emerging hiPSC colonies (D10). TRA-1-60 live staining confirms the expression of this marker by newly formed hiPSC colonies by fluorescence microscopy (D11; scale bar $=100 \mu \mathrm{m}$ ). (c) Top: representative image of immunofluorescence analysis of a whole culture chamber after 14 days of fibroblast reprogramming. The whole figure was obtained by tiling single microscopy images next to each other ( scale bar $=1 \mathrm{~mm}$ ). Bottom: enlargement of the region highlighted by the red frame in the whole chamber, assayed for the indicated markers (scale bar $=100 \mu \mathrm{m})$. (d) Dot plot indicating the number of hiPSC colonies obtained in each independent microfluidic culture chamber from the indicated fibroblast cell lines. The superimposed red error bars represent mean \pm standard deviation of the data. The number of replicates, $n$, is also indicated for each condition. (e) Top: representative image of immunofluorescence analysis of TRA-1-60 in a culture chamber where fibroblasts were reprogrammed in presence of Boost Supplement II for 14 days (scale bar $=1$ $\mathrm{mm}$ ). Bottom: enlargement of the region highlighted by the red frame in the top image, assayed for the indicated markers (scale bar $=100 \mu \mathrm{m}$ ). 
a
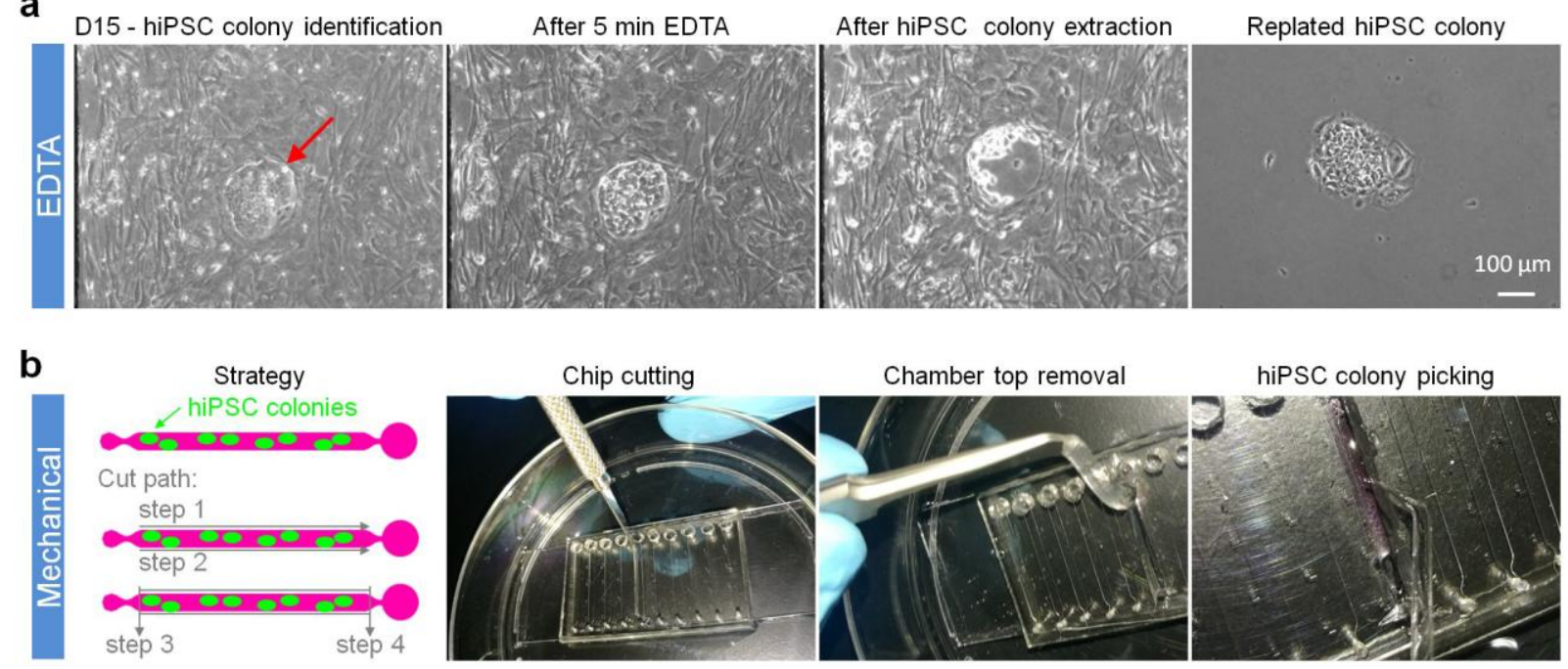

hiPSC colony picking

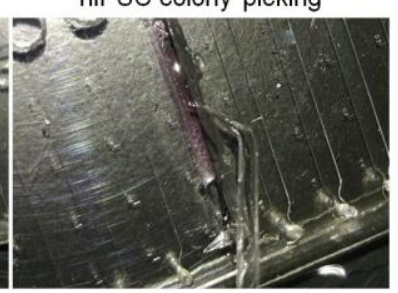

Figure 5 | Methods of hiPSC colony extraction from the microfluidic culture system. (a) Isolation of hiPSC colonies from a single culture chamber as a pool by EDTA treatment, and replating in a standard dish. (scale bar $=100 \mu \mathrm{m}$ ). (b) Procedure for single hiPSC colony mechanical picking from a chamber of the microfluidic device. See also Supplementary Video 2. 